\title{
The effect of the impact of Comet Shoemaker Levy-9 on Jupiter's Aurorae
}

\author{
S. Miller, ${ }^{1}{ }^{2}$ N. Achilleos, ${ }^{1}{ }^{2}$ B.M. Dinelli, ${ }^{13}$ H.A. Lam, ${ }^{1}$ J. Tennyson, ${ }^{1}$ M.-F. \\ Jagod, ${ }^{4}$ T.R. Geballe, ${ }^{5}$ L.M. Trafton, ${ }^{6}$ R.D. Joseph, ${ }^{7}$ G.E. Ballester, ${ }^{8}$ K. \\ Baines, ${ }^{2}$ T.Y. Brooke ${ }^{9}$ and G. Orton. ${ }^{2}$
}

\begin{abstract}
We present infrared spectra and images of the jovian aurorae taken at wavelengths sensitive to the $\mathrm{H}_{3}^{+}$molecular ion during the period around the impact of Comet Shoemaker Levy-9. The spectra were obtained using CGS4 on the United Kingdom Infrared Telescope and the images using NSFcam on NASA's Infrared Telescope Facility. Comparison with spectra obtained in May, 1993, shows that while the relative intensities of the northern and southern auroral zones prior to and during impact week (July $16-22,1994$ ) were broadly comparable with those of 1993 , a few days after the last collision the northern aurora was considerably enhanced and its southern counterpart somewhat depressed. The north/south auroral ratio was returning to more normal values a week later. The effect of material drifting from the impact sites to the southern auroral zone is discussed in relation to these results.
\end{abstract}

\section{Introduction}

Much information concerning the effects of the impacts of the twenty-plus fragments of Comet Shoemaker Levy-9 (SL9) has already been made available both to the scientific community and the public at large. One region of the jovian atmosphere which was likely to be affected by the impacts was the outer layer, which includes the ionosphere. Observation of this region during the impacts was limited, but interesting data are emerging (Ballester et al, 1994; Clarke et al., 1994; Encrenaz et al., 1994; Orton et al., 1994). In recent years, the molecular ion $\mathrm{H}_{3}^{+}$has proved an invaluable probe of the physical conditions in the aurorae and general ionosphere (see review by Miller et al., 1994) and

\footnotetext{
${ }^{1}$ University College London, London, U.K.

${ }^{2}$ Visiting astronomers at the NASA IRTF.

${ }^{3}$ Istituto di Spettroscopia Molecolare, Bologna, Italy.

${ }^{4}$ University of Chicago, U.S.A.

5 JACH, Hilo, Hawaii, U.S.A.

${ }^{6}$ University of Texas, Austin, U.S.A.

${ }^{7}$ Institute for Astronomy, Honolulu, Hawaii, U.S.A.

${ }^{8}$ University of Michigan, Ann Arbor, U.S.A.

${ }^{\ominus}$ Jet Propulsion Laboratory, Pasadena, U.S.A.
}

\section{Copyright 1995 by the American Geophysical Union.}

\section{Paper number 95GL00700 \\ 0094-8534/95/95GL-00700\$03.00}

the morphology of the auroral zones (Connerney et al., 1993). Here we present a preliminary analysis of the effect of the SL9 collisions on the auroral zones of Jupiter, as monitored by variations in the emission of this ion. Spectra were obtained as part of the United Kingdom Infrared Telescope's SL9 impact programme; images were obtained as part of the effort of the NASA Infrared Telescope Facility Comet Collision Science Team.

\section{Observations}

During the SL9 impact period, spectra of the auroral regions were obtained using the UKIRT CGS4 long slit spectrometer at both moderate $(\lambda / \Delta \lambda=1000)$ and echelle $(\lambda / \Delta \lambda=15000)$ resolving powers. The combination of the $300 \mathrm{~mm}$ focus camera and the 75 lines $/ \mathrm{mm}$ grating gave a pixel size of 1.54" x 1.54" at moderate resolution. For the echelle spectra a slit width of 1.2 " was used, with the individual pixels extending 2" along the slit. At that time, 1" was equivalent to about 3,700 kilometers at Jupiter. The spectra presented here were obtained on July $15,17,19,20,25$ and 27 , with the spectrometer slit aligned from pole-to-pole along the central meridian. Different central meridian longitudes ( c.m.l.; all longitudes are expressed in System III) could thus be sampled as Jupiter rotated under the slit.

At moderate resolution, spectra were obtained around a central wavelength of $3.45 \mu \mathrm{m}$ and $4.00 \mu \mathrm{m}$; at echelle resolution spectra were obtained at a central wavelength of $3.533 \mu \mathrm{m}$. The spectral range was approximately $0.25 \mu \mathrm{m}$ at moderate resolution and $0.017 \mu \mathrm{m}$ at echelle. Typical integration times were 5-10 seconds, with total integrations per complete spectrum between one and several minutes. Each spectrum was sampled every $1 / 3$ resolution element. The reference sky position was 120 " perpendicular to the slit orientation. Flux calibration and removal of atmospheric features were carried out using the standard star BS5056. The reduced spectra were compared to CGS4 spectra of Jupiter obtained during May 3 - 5, 1993 (Lam et al., 1994).

Images sensitive to $\mathrm{H}_{3}^{+}$auroral emissions were obtained using the NASA IRTF facility camera, NSFcam. This has a $1 \%$ circular variable filter (c.v.f.) which enables a continuous range of wavelengths to be selected. The platescale used throughout gave pixels subtending 0.35 " $\times 0.35$ ". The images used here were obtained with the c.v.f. centred at $3.4 \mu \mathrm{m}$; 1s-sky subtracted exposures were co-added to give total exposure times of 60 s per image. Flux calibration made use of standard 
star BS5530. Because the dome was too bright for the sensitive InSb array in NSFcam, sky frames were used for flat fielding. Images were obtained on July 15, 17, 27 and 28 and August 7 at a number of different c.m.l.s. For both the spectra and images, the overall seeing was around 1" as a result of atmsopheric conditions and having to observe during the evening.

\section{Results}

The relative brightness of the northern and southern auroral zones depends on the c.m.l. at which the measurements are made. Variations are due in part to the exact geometry at which the auroral arcs are viewed, in part to temporal effects and in part to intrinsic longitudinal dependence. Typically the northern aurora is brighter for c.m.l.s between 150 and 210, with the southern brighter between 330 and 50 , and a mixed, intermediate situation otherwise. Normal N/S total auroral flux ratios vary between 0.3 and 3 (Lam, 1995). For example, in data obtained between May 1 and 3, 1993 , the N/S ratio for c.m.l.s between 40 and 50 was $0.35 \pm 0.08$; between 160 and $210, \mathrm{~N} / \mathrm{S}$ had an average value of 1.83, ranging between 3.0 (at 160) and 1.3 (at 210) (Lam et al., in preparation).

Figure 1 shows a plot of N/S auroral ratios measured during the period July 12 to August 7, 1994. Data from CGS4 spectra and NSFcam images are combined on the graph. Errors are shown by the length of the data bar. The 40-plus observations represent a good longitudinal sample of the planet, and full details - too extensive to publish here - are available from the authors on request. The data from the images were obtained straightforwardly by measuring the total flux visible in each of the individual auroral regions and calculating the ra-

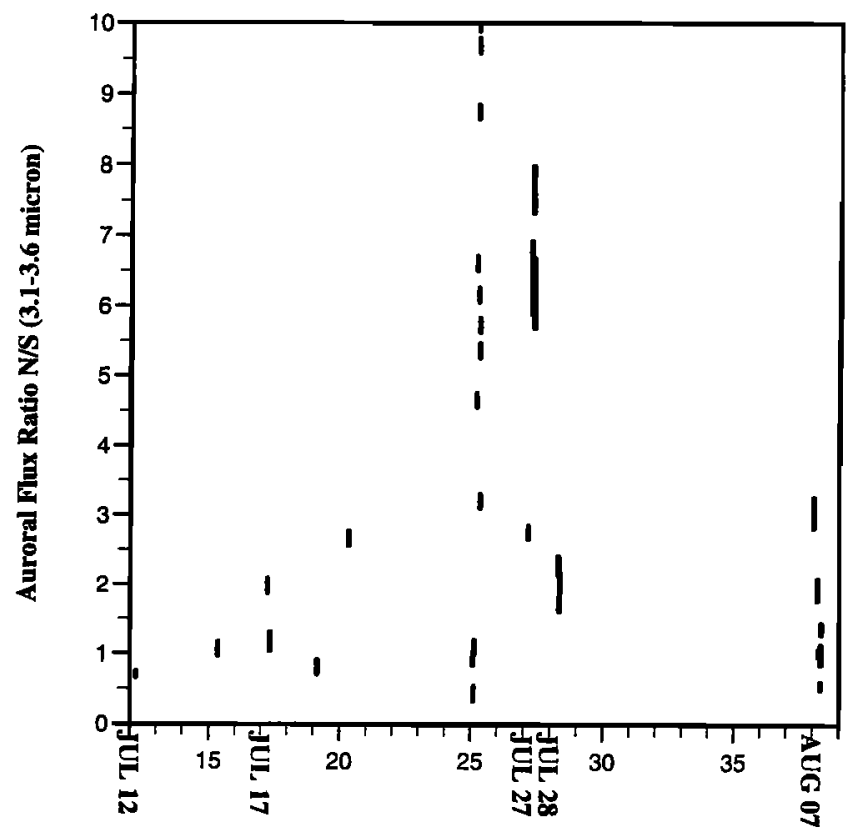

Figure 1. North/south auroral intensity ratios during the SL9- Jupiter Impact period. tio. At $3.4 \mu \mathrm{m}$, the continuum emission contributed less than $10 \%$ of the total $\mathrm{H}_{3}^{+}$output.

The process used to obtain ratios for the spectra was more complicated, however. Emission lines of the $\mathrm{H}_{3}^{+}$ $\nu_{2}$ band dominate the spectra in both auroral zones, and extend across the planet at all latitudes at a much reduced intensity. The fits to the spectra were obtained using a combination of laboratory $\mathrm{H}_{3}^{+}$wavelengths and ab initio Einstein A coefficients for the individual transitions (Kao et al. 1991). We attempted to derive independent column densities $(\rho)$ and temperatures for each individual spectral row. Typically, densities of a few tens $\times 10^{12} \mathrm{~cm}^{-2}$ are obtained with temperatures in the region of 800 to $1000 \mathrm{~K}$. However these parameters are strongly ( $>99 \%$ ) correlated and for the rest of this paper we make use of an $\mathrm{H}_{3}^{+}$comparison emission parameter, E(c.m.l.), computed as follows.

The best-fit values of $\rho$ and T were obtained for each row in each of the northern and southern auroral zones showing bright auroral emission. For the value of $\mathrm{T}$ obtained, the total emission per molecule of $\mathrm{H}_{3}^{+}$was calculated assuming quasi-thermal equilibrium (Kim et al. 1992). This was then multiplied by the column density and the results for the bright rows added for each zone and multiplied by the distance subtended by the pixel length on Jupiter. This gave a result in ergs $\mathrm{s}^{-1}$ $\mathrm{sr}^{-1} \mathrm{~cm}^{-1}$ and was equivalent to measuring the flux per steradian coming from a $1 \mathrm{~cm}$-wide strip along the c.m.l. on Jupiter, spanning the auroral region in question. This process made knowledge of the pixel filling factor unnecessary and made it possible not only to obtain the north/south brightness ratio in each spectral image, but to compare with the 1993 data on an absolute scale.

Figure 1 shows that while data collected prior to and during impact week have N/S auroral ratios similar to those normally pertaining, on July 25 and July 27 the peak N/S ratios reached ten and eight respectively, much higher than normally measured and well outside of the range of error of the observations. By August 7 , however, the situation was returning more or less to normal. That the $\mathrm{N} / \mathrm{S}$ enhancement is not simply due to viewing geometry can be demonstrated by comparing ratios for longitudinal regions for which we have several measurements. For instance, in the 1993 dataset, c.m.l 210 has N/S ratio of 1.3 and c.m.l. 2410.8 . On July 15 , at c.m.l. 223 , we measure $\mathrm{N} / \mathrm{S}=1.1$, consistent with the previous year. But on July 25, at c.m.l. 236, we find $\mathrm{N} / \mathrm{S}=5.7$ and on July 27 , at c.m.l. $224, \mathrm{~N} / \mathrm{S}=$ 6.3 , both clearly enhanced. By August 7, N/S at c.m.l. 230 has fallen back to 2.3. Other longitudinal regions show similar effects. These data raise the question as to whether the high N/S ratio was due to the enhancement of the northern zonal emission, the suppression of the south or a combination of the two.

Observing conditions made it difficult to obtain reliable absolute fluxes for the images. The higher resolution spectra could be calibrated, however, giving reliable values of $\mathrm{E}$ (c.m.l.). In Figure 2, these are presented for the data of July 25 and July 27, 1994 and compared 

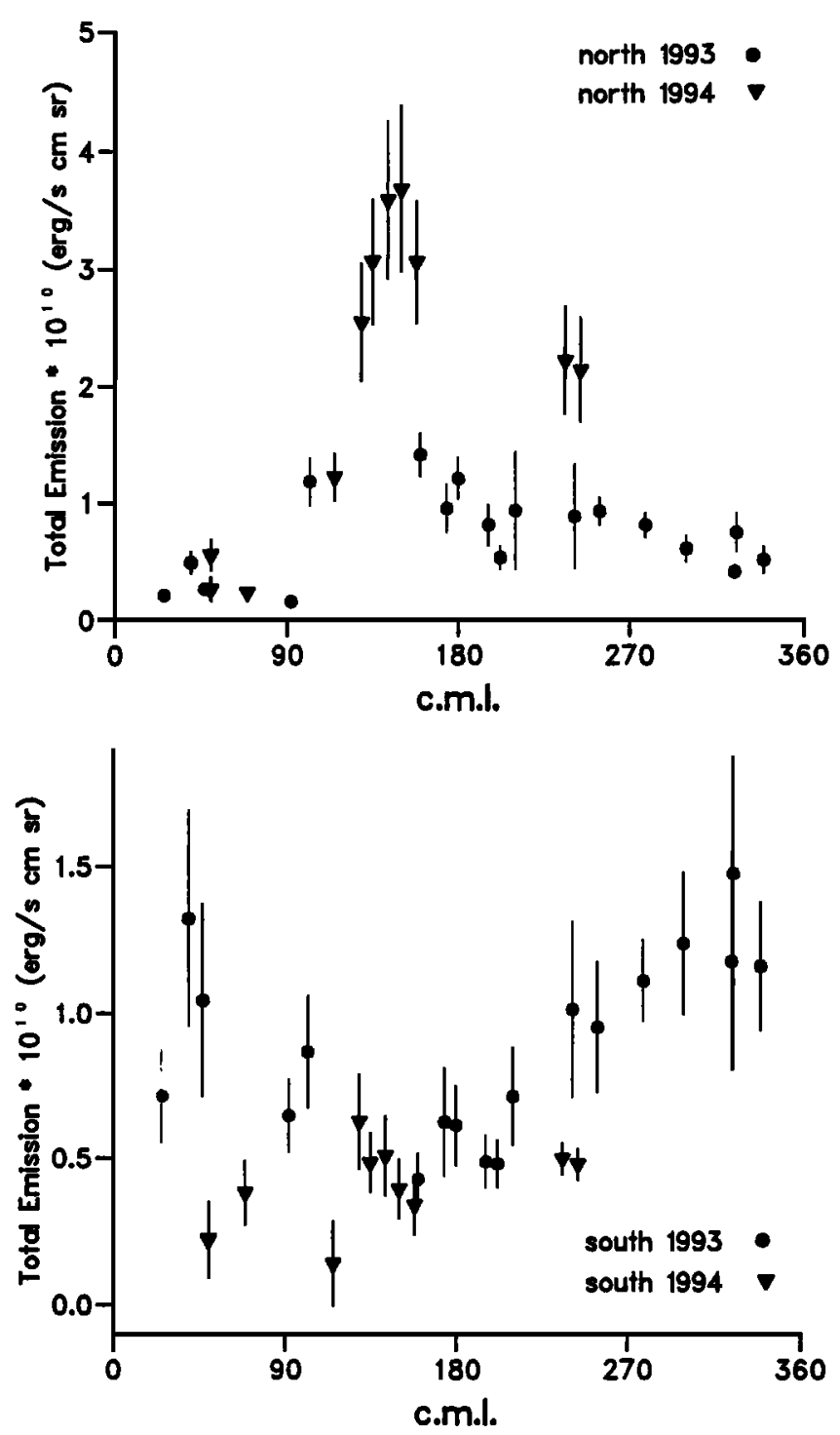

Figure 2. Total $\mathrm{H}_{3}^{+}$emission, E(c.m.l.) as a function of c.m.l. in (top) northern auroral region and (bottom) southern auroral region.

with those for May $3-5,1993$. The 1993 data show that maximum values of $\mathrm{E}(\mathrm{c} . \mathrm{m} . \mathrm{l}$.) in both the north and the south were around $1.5 \times 10^{10} \mathrm{erg} \mathrm{s}^{-1} \mathrm{sr}^{-1} \mathrm{~cm}^{-1}$. The 1994 data, on the other hand, indicate a dramatic change. In the south, maximum auroral emissions are suppressed to less than $7 \times 10^{9} \mathrm{erg} \mathrm{s}^{-1} \mathrm{sr}^{-1} \mathrm{~cm}^{-1}$, while in the north they reach values well in excess of $3 \times 10^{10}$ erg s $\mathrm{s}^{-1} \mathrm{sr}^{-1} \mathrm{~cm}^{-1}$.

In the south, on July $25 / 27$ the suppression is greatest at those c.m.l.s which normally show the brightest emission; at the lower emission longitudes the situation differs little from 1993. In the north, the lower emission regions on July $25 / 27$ are similarly not much different from 1993. It is the normally bright auroral region around c.m.l. 150-180 which is enhanced by up to a factor of three. This combination of northern enhancement and southern suppression thus accounts for the N/S ratio close to 10 , observed at c.m.l.s around 150, which shows up in Figure 1.

\section{Discussion}

Clarke et al. (1994) noticed an unusual auroral event linked with the impact of one of the larger fragments of SL9. Fragment $\mathrm{K}$ caused an auroral brightening about an hour after impact at a northern latitude of around 44 and at a point magnetically conjugate to the impact site. Dessler and Hill (1994) have suggested that this could have been due to ions, forced onto field lines in the south by the impact itself, precipitating in the northern hemisphere. In the absence of an impact, however, this mechanism does not appear to be appropriate for our results. Unrelated to the SL9 impacts, bright ultraviolet events within the normal auroral ovals, lasting up to a few hours, have been observed (Ballester work in progress, 1994). But these have indicated positive correlation between the northern and southern emission intensities, not the anti-correlation noted here.

One possible explanation for our results is that high altitude particulates - or molecules (e.g. water; Cravens 1994) - from the impact sites drifted southwards into the ionosphere in the auroral regions. We calculate that drift speeds between 50 and $100 \mathrm{~m} \mathrm{~s}^{-1}$ would be required for the particulates to cover the required distance in time to have the observed effect. This is not unreasonable; Clarke et al. (1994) observed material drifting northwards at similar speeds. Impact site material would have lowered the conductivity of the southern auroral zone, reducing the current flowing there, cooling the southern auroral region and leading to lower $\mathrm{H}_{3}^{+}$ emission. The field lines from the auroral zones couple to the equatorial plasma sheet, effectively transferring momentum from the planet to the plasma to maintain partial co-rotation via the current flowing in the ionosphere (Strobel and Atreya, 1983). A current reduction in the south would reduce this effect, allowing the equatorial plasma to slip relative to the planet, in turn, increasing the electric field in the northern auroral region and the current flowing there. The resulting additional Joule heating of the northern auroral ionosphere would increase $\mathrm{H}_{3}^{+}$emission there.

The high correlation of column density and temperature in our fits makes it impossible to separate the two parameters. But if we assume (i) a typical auroral temperature around $900 \mathrm{~K}$ and (ii) no change in column density, the effects we see would be produced by a lowering of the peak southern auroral temperature by about $100 \mathrm{~K}$ coupled with a temperature increase in the north of the same magnitude. This leads us to estimate that a $25-40 \%$ reduction in southern conductivity is needed to produce the effects we observe, depending on assumptions about the plasma disk conductivity. Further modelling is underway (Achilleos, work in progress, 1994) to see if this mechanism could account in detail for the observed suppression of the bright southern regions/enhancement of the bright northern regions.

\section{Conclusion}

We have noted a clear anti-correlation in northern and southern auroral $\mathrm{H}_{3}^{+}$emissions peaking some three 
to five days after the last impact and appearing to relax towards normal over the next few days. To our knowledge, this is the first time that such an anti-correlated event has been noted and it would therefore appear to be associated with the aftermath of the SL9 collision. Timescales for the transport of impact site material seem to fit the requirements for our results and we have proposed a model by which this might produce the observed effects. But further calculations are clearly required before we can make a more definite statement as to the precise mechanism.

Acknowledgments. It is a pleasure to acknowledge the help and advice of Professor T. Oka of the University of Chicago. This paper has also benefitted considerably from comments by the referees and editorial staff of GRL. We would also like to thank the staff at both UKIRT and IRTF for their great efforts to ensure that the SL9 collision observing campaign was a success. UK PPARC and US NSF and NASA supported this work financially.

\section{References}

Ballester G.E. et al., Geophys. Res. Lett. (submitted) 1994. Clarke J. et al., Hubble Space Telescope Far UltraViolet . Images of Jupiter during the impacts of Comet Shoemaker Levy-9, Science (submitted) 1994.

Connerney J.E.P., R. Baron, T. Satoh and T. Owen, Images of $\mathrm{H}_{3}^{+}$at the Foot of the Io Flux Tube in Jupiter's Atmosphere, Science, 262, 1035, 1993.

Cravens T.E., Comet SL 9 Impact with Jupiter: Aeronomical Predictions, Geophys. Res. Lett., 21, 11, 1994.

Dessler A. and T.W. Hill, Effects of Comet Impacts on the Jovian Magnetosphere, Bull. Am. Astron. Soc., 26, 05.09, 1994.

Encrenaz Th., R. Schulz, J.A. Stuewe, G. Widerman, P. Drossart and J. Crosisier, Near-IR Spectroscopy of Jupiter at the time of SL9 Impact using NTT-IRSPEC, The Messenger, 77, 40, 1994.

Kao. L., T. Oka, S. Miller and J. Tennyson, A Table of
Astronomically Important Transitions of $\mathrm{H}_{3}^{+}$, Astrophys J. Suppl., 77, 317, 1991.

Kim Y.K., J.L. Fox and H.S. Porter, Densities and Vibrational Distribution of $\mathrm{H}_{3}^{+}$in the Jovian Auroral Ionosphere, J. Geophys. Res., 97, 6093, 1992.

Lam H.A., S. Miller, J. Tennyson, T.R. Geballe, L.M. Trafton and G.E. Ballester, Mapping the Jovian Ionosphere using $\mathrm{H}_{3}^{+}$as a Probe, Bull. Am. Astron. Soc., $26,05.28,1994$.

Lam H.A., PhD. Thesis, University College London, 1995.

Miller S., H.A. Lam and J. Tennyson, What astronomy has learned from observations of $\mathrm{H}_{3}^{+}, J$. Can. Phys. in press, 1994.

Orton G. et al., The NASA Infrared Telescope Facility Investigation of Comet Shoemaker Levy-9 and its collision with Jupiter: Preliminary Results, Science, (submitted), 1994.

Strobel D. and S. Atreya, Ionosphere, in Physics of the Jovian Magnetosphere, ed. A.J. Dessler, 51(Cambridge University Press) 1983.

N. Achilleos, B.M. Dinelli, H.A. Lam S. Miller and J. Tennyson, Department of Physics and Astronomy, University College London, Gower Street, London WC1E 6BT, U.K.

M.-F. Jagod, Department of Chemistry, University of

Chicago, S. Ellis Ave., Chicago, Illinois 60637, U.S.A.

T.R. Geballe, UKIRT, Joint Astronomy Centre,

Komohana Street, Hilo, Hawaii 96720, U.S.A.

L.M. Trafton, McDonald Observatory, University of

Texas at Austin, Austin, Texas 78712, U.S.A.

R.D. Joseph, Institute for Astronomy, University of

Hawaii, Woodlawn Drive, Honolulu, Hawaii 96822, U.S.A.

G.E. Ballester, Department of Atmospheric, Oceanic and Space Sciences, University of Michigan, Ann Arbor, Michigan 48109-2143, U.S.A.

K.H. Baines, T.Y. Brooke and G.S. Orton, Jet

Propulsion Laboratory, California Institute of Technology, 4800 Oak Drive, Pasadena, California 91109, U.S.A.

(received December 9, 1994; revised February 8, 1995; accepted February 13, 1995.) 